\title{
RADIATION EXPOSURE AND THYROID CANCER RISK AFTER THE FUKUSHIMA NUCLEAR POWER PLANT ACCIDENT IN COMPARSION WITH THE CHERNOBYL ACCIDENT
}

\author{
Shunichi Yamashita1,2,*, Noboru Takamura ${ }^{3}$, Akira Ohtsuru²,4, Shinichi Suzuki²,5 \\ ${ }^{1}$ Department of Radiation Medical Sciences, Atomic Bomb Disease Institute, Nagasaki University \\ ${ }^{2}$ Radiation Medical Science Center for the Fukushima Health Management Survey, Fukushima Medical \\ University \\ ${ }^{3}$ Department of Global Health Medicine and Welfare, Atomic Bomb Disease Institute, Nagasaki University \\ ${ }^{4}$ Department of Radiation Health Management, Fukushima Medical University \\ ${ }^{5}$ Department of Thyroidology and Endocrinology, Fukushima Medical University \\ Running title: Thyroid cancer in Fukushima
}

Received month date year, amended month date year, accepted month date year

\begin{abstract}
The actual implementation of the epidemiological study in human health risk from low dose and low-dose rate radiation exposure and the comprehensive long-term radiation health protection are important especially after the radiological and nuclear accidents because public fear and concern about the long-term health effects of low dose radiation exposure have increased considerably. Since the Great East Japan earthquake and the Fukushima Daiichi Nuclear Power Plant accident in Japan, Fukushima Prefecture has started the Fukushima Health Management Survey Project for the purpose of long-term health care administration and medical early diagnosis/treatment for the prefectural residents. Especially on a basis of the lessons learned from the Chernobyl accident, both thyroid examination and mental health care are critically important irrespective of the level of radiation exposure. There are considerable differences between Chernobyl and Fukushima regarding radiation dose to the public, and it is very difficult to estimate retrospectively internal exposure dose from the short-lived radioactive iodines. Therefore, the necessity and intermediate results of thyroid ultrasound examination in Fukushima targeting children will be reviewed and discussed in order to avoid any misunderstanding and misinterpretation of a high detection rate of childhood thyroid cancer in Fukushima.
\end{abstract}

\section{INTRODUCTION}

The scientific understanding about the relationship between radiation exposure dose and health risk is needed to apply any countermeasure against radiological and nuclear accidents. According to the accumulated data from the survivors of the Atomic bomb analyzed by the Radiation Effects Research Foundation, risks of leukemia and solid cancers have been proved in a dose-dependent manner with a certain latency period ${ }^{(1,2)}$. Radiation-associated human cancer may arise not only after external exposure but also due to internal exposure to radioactive iodine which can increase risk of thyroid cancer as epidemiologically clarified just after the Chernobyl accident, and both factors become especially important to understand the health effects ${ }^{(3)}$. Low dose radiation exposure and the risk of thyroid cancer should be carefully evaluated learning from the recent progress in radiation biology and oncology(4).

After the 2011 Fukushima accident, there was widespread environmental contamination with radioactive nuclides. This accident again highlighted issues regarding countermeasures, including iodine thyroid blocking, since suitable medication with stable iodine is effective for reduction and prevention of the internal exposure to radioactive iodine immediately before or after an accident ${ }^{(5)}$, and the safety control of food restricting consumption of contaminated milk and other products need to be put into practice after that. Although the side effects and effectiveness of iodine tablet dosage needs to be verified ${ }^{(6)}$, appropriateness of actual implementation of iodine thyroid blocking in Fukushima should be further evaluated. The outline on the nuclear accident in Fukushima and thyroid cancer risk will be summarized, assuming the possibility of the initial exposure to radioactive iodine, and drawing lessons from the Chernobyl nuclear power plant accident, referring our previous review $\operatorname{article}^{(7)}$.

\section{LESSONS LEARNED FROM CHERNOBYL}

Chernobyl was of outstanding importance for the issues arising after the Fukushima nuclear power plant accident. We, therefore, at first summarize these lessons before focusing on exposures after Fukushima

\footnotetext{
*Corresponding author: Shunichi Yamashita, MD, PhD. 1-124 Sakamoto, Atomic Bomb Disease Institute, Nagasaki University Nagasaki 8528523, Japan, E-mail: shun@nagasakiu.ac.jp, Tel: 095-819-7116, Fax: 095-819-7117 (Director of the WHO-REMPAN Collaborating Center at Nagasaki University
} 
and the situation regarding thyroid ultrasound examination.

Large-scale release of radioactive materials continued until May 61986 after the Chernobyl nuclear power plant accident in the early morning of April 26, 1986. The main radioactive materials emitted into the environment were iodine-131, cesium-134, 137, niobium-95, cerium-144, ruthenium-103, 106, strontium-90, and plutonium-239 and 240, which reached a total amount of 14 exabecquerel $\left(\times 10^{18}\right)$ and other radioactive materials were widely diffused in the Northern Hemisphere around Europe ${ }^{(8)}$.

In particular, the critical problems for the local residents has been that iodine-131 was found in milk from cows that had been feeding on contaminated grass from areas close to Chernobyl. Due to an initial lack of knowledge of the necessity of restriction of contaminated milk and then inappropriate distribution and insufficient restriction of the contaminated milk consumption by the government officials, people continued to ingest iodine-131, particularly children of Belarus, Russia, and Ukraine of the former USSR at the time of the accident. Iodine has the property to be selectively taken in by the thyroid gland, which also applies to iodine-131. In addition, Chernobyl is an inland area and an area that lacks iodine, which became the contributive factor exacerbating internal exposure of the thyroids of children who ingested iodine-131 contaminated milk. These children were exposed to an estimated dose of tens to several thousand millisievert of thyroidal radiation. As a result, infant thyroid cancer increased rapidly in people who were children (especially 0 5 years old) at the time of the accident ${ }^{(9)}$. A case-control study has demonstrated a positive relationship between childhood thyroid cancer occurrence and thyroidal iodine-131 internal exposure dose $\mathrm{e}^{(10)}$. Although there was a clear increase in childhood thyroid cancer, it was not easy to scientifically demonstrate dose response relationship in a case-control study. However, there are model-based assumptions using linear non-threshold (LNT) model $^{(11)}$ about significant risk elevation at doses of 50 200 mSv, or above ${ }^{(12-14)}$. Aside of the difficult challenge of thyroid dose re-evaluation, the comparative study in children who were born before and after the Chernobyl accident also supports the etiological role of short half-lived radioactive iodine on childhood thyroid cancer despite no direct measurement of thyroid exposure dose ${ }^{(15)}$. The number of thyroid cancers continues to increase even 25 years after the accident ${ }^{(16)}$, and has amounted to about 6,000 people. The peak of the infant thyroid cancer observed from 1991 to 1996; at present all exposed patients are adults. Although detailed molecular mechanism of thyroid carcinogenesis is being examined, the clear radiation-associated signature genes have not been proven $^{(17)}$. Inherited susceptibility has been also examined in a genome-wide association study of
Chernobyl thyroid cancer $^{(18)}$, which did not reveal genetic variants specifically associating with thyroid cancer arising after radiation exposure as compared to those underlying predisposition to sporadic thyroid cancer in the European population ${ }^{(19)}$.

\section{RADIATION DOSE ESTIMATION AFTER THE FUKUSHIMA NUCLEAR POWER PLANT ACCIDENT}

All the nuclear reactors of the $1^{\text {st }}$ and $2^{\text {nd }}$ TEPCONuclear Power Plants in Fukushima stopped automatically after the Great East Japan Earthquake on March 11, 2011. However, all the power supplies of reactor No.1 4 for cooling were lost due to the earthquake and tsunami, hydrogen explosion happened in succession and as a result, a lot of radioactive materials were emitted to the outside environment, and spread through the wind.

According to the environmental measurement data in Fukushima, radioactive material dispersed through the wind after the hydrogen explosion of the nuclear power plant, and contaminated the surface. The main radioactive nuclide emitted from the nuclear power plant was iodine-131 with short physical half-life of about 8 days, and the area with high spatial dose rate at that time showed immediate declining trend. Among the radioactive nuclides emitted from the same nuclear power plant, cesium-134 and 137 have long physical half-life, and deposit in soil, on roofs, on the outer walls of the buildings, etc. for a long time, and so the risk of childhood thyroid cancer was confused at first to be caused by external exposure ${ }^{(20)}$. Shipment and consumption restrictions on food, concerning radioactive iodine and the amount of cesium, began with the milk of Fukushima Prefecture and the spinach of Fukushima, Ibaraki, and Tochigi Prefectures on March 21. The safe interim standard value on food, which causes a maximum annual internal exposure dose of $5 \mathrm{mSv}$, was set in the end of March after the accident, and the shipment restriction and ingestion restrictions on food exceeding the target value were conducted. As of April 2012, a lower annual internal exposure dose of $1 \mathrm{mSv}$ was set in order to respond the pubic request of safer restriction.

Unfortunately, based on the weather survey data, radiation data, and the information on radioactive material discharge immediately after the accident, wind velocity and SPEEDI (System for Prediction of Environmental Emergency Dose Information), which was scheduled to predict and calculate the air concentration of a radioactive material, radiation dose, etc., could not operate due to insufficient information on the source of emission. In many cases, the actual external exposure dose was quite low, however, due to the shielding effect of the buildings. 


\section{SHORT TITLE}

The estimation of the external radiation exposure dose in people residing in Fukushima at the time of the earthquake is currently being conducted as the basic investigation for prefectural health management survey by Fukushima Prefecture ${ }^{(21)}$. The study protocol for this survey was published elsewhere ${ }^{(22)}$. According to the data from the original basic survey targeting the residents of the evacuation prepared zone, maximal estimated external exposure dose among 9,747 people (excluding the radiation operation workers) of Kawamata-machi (Yamakiya area), Namie-machi, and Iitate-mura, which was carried out as a pilot investigation for 4 months until July 11, 2011, was 23 mSv. Thus, $94.6 \%$ of residents were estimated to be exposed to less than $5 \mathrm{mSv}$ per year, and 99.3\% were under $10 \mathrm{mSv}$. The most recent data released from Fukushima Prefecture on August 2014 indicated that the average dose of more than 450,000 residents during 4 months is below $1 \mathrm{mSv}$. Fukushima residents' health management survey committee evaluated these results as "it is difficult to consider the health impairment caused by radiation". Although the situation regarding the possibility of radiation-related health risk remains unclear, health management and efforts for continuous reduction of future radiation exposure, such as decontamination and avoidance of contaminated foods, are required.

On the other hand, children in Iitate-mura and Iwaki prefecture have been predicted with possible thyroid exposure which may reach $100 \mathrm{mSv}$ by SPEEDI, even though they were outside the 20-km range. According to the report on the thyroid internal exposure estimation, which was conducted from March 26 to 30 just after the nuclear power plant disaster, the equivalent dose of $100 \mathrm{mSv}$ was not confirmed. From this result, any increase in thyroid cancer outside the evacuation area would hardly be expected. Yet, according to the report by Hirosaki University ${ }^{(23)}$, equivalent dose for the thyroid might have reached $10 \mathrm{mSv}$ in the infants who stayed within the range of $20 \mathrm{~km}$ at the time of the accident; undoubtedly it is necessary to observe these individuals for a long period of time. Direct measurement of internal exposure in the very early period after the accident suggests quite a low possibility of any stochastic health effect ${ }^{(24)}$.

WHO released the estimates of exposure doses around Fukushima in May 2012. By applying SPEEDI, based on conservative assumption (i.e. without taking refuge for four months after the accident in the evacuation prepared area and limiting the consumption of the food that were restricted for shipment and ingestion), the prediction data are calculated from a viewpoint of protection on the assumption of overestimation ${ }^{(25)}$. According to the report, a 1 year-old child's thyroid equivalent dose is $10 \sim 100 \mathrm{mSv}$ in Minami-soma, Iwaki, Iitate-mura, prepared evacuation area, and 1 10 mSv in prefectures adjacent to Fukushima. However, it should be noted that the actual values based on direct measurements as outlined above are markedly lower.

\section{THYROID ULTRASOUND EXAMINATION IN FUKUSHIMA}

At and during the time when the thyroid exposure dose was unknown, in order to overcome radiation fear and unexpected future risk of late-occurrence of thyroid cancer in children and also to respond the strong request to screen all the children's thyroid gland, the thyroid ultrasound examination was carefully planned and implemented 7 months after the accident. In addition to the basic investigation of dose estimation in Fukushima, the following: 1) thyroid ultrasound examination, 2) health checkup, 3) examination regarding the mental health performance and lifestyle, and 4) the examination of expecting and nursing mothers, have been commenced and their progress has been uploaded at the homepage of Fukushima Medical University ${ }^{(26)}$. From the results of the environmental radiation dose estimates and thyroid radiation dose investigation, there are very few health effects, if any, that might be expected. However, thyroid ultrasound examination was started from October 2011 for approximately 370,000 people 18 years or younger, based on the knowledge of the rise in thyroid cancer risk in a group exposed to radiation through radioactive iodine ingestion during childhood in Chernobyl. As a result, the initial thyroid ultrasound examination of approximately 38,000 people among 48,000 candidates (approximately 80\%) of the evacuation prepared zone was finished by March 2012, and the examination area was expanded sequentially from Fukushima city after May 2012. Diagnostic criteria and protocol are introduced and evaluated by the external committee by thyroid specialists through cooperation with associated academic societies to make a decision regarding the need of precision management and image evaluation of the thyroid ultrasound diagnosis and a secondary examination. Most were judged as normal but recognized examples of minute node, benign findings (cyst, etc.) exist, and the standardization of the diagnostic imaging and observation process was also attained ${ }^{(27)}$. The findings regarded as suspicious that require secondary examination (a detailed ultrasound examination, blood test and urinalysis, cytological diagnosis, etc. as needed) was about $0.5 \%$. Although minute change such as the A2 finding is detected at a relatively high frequency due to the improvement in ultrasound diagnostic technology, it is necessary to pay attention to the change with qualitative diagnosis over time. Hereafter, medical examination based inside and outside the prefecture is organized by introduction and accuracy control of these criteria, and the thyroid examination for all the persons fulfilling the inclusion criteria will be performed over next two years until the 


\section{SAMPLE ET AL}

age of 20 years old, without compulsion. Even if the fixed target group of approximately 370,000 people is over 20 years old, medical examination every five years is planned by their free will, and so it is more important how to keep the participation rate of examination and follow up the target person periodically for more than 30 years.

As predicted, the massive thyroid ultrasound screening has brought about an increased detection rate of childhood thyroid cancer in Fukushima, which ironically exaggerates radiation fear and concern, especially for patients' parents because of inappropriateness and artlessness of public risk communication and also due to the lack of understanding of thyroid cancer biology and epidemiology by the public.

In contrast to the uncertainty of internal exposure and thyroid cancer risk in Fukushima, the survivors of the atomic bombings were mainly exposed to an instant external radiation, which led to an increased risk of the solid tumors after a 10-year latency, and we reacknowledged the "statistical" cancer risk at the doses higher than $100(\sim 200) \mathrm{mSv}^{(28)}$. Furukawa et al. has just reported significant dose-response relationship between externally exposed organ-dose and thyroid cancer risk at the level higher than $150 \sim 200 \mathrm{mSv}^{(29)}$. There are a big difference in radiation dose and the mode of exposure between the atomic bomb survivors in Hiroshima and Nagasaki, Chernobyl victims and the people in Fukushima. Therefore, although the situation regarding the possibility of low-dose rate radiationrelated health effects remains unclear in Fukushima, the future increase of radiation-associated thyroid cancer risk has not been predicted.

In contrast to the initial increase of childhood thyroid cancer 4 5 years after the accident in Chernobyl, where it was mainly due to internal exposure to radioactive iodine, the first precedent thyroid examination conducted now in Fukushima is considered to be the check of the underlying disease frequency (baseline incidence) of the thyroid gland under the condition of screening before the effect of the nuclear power plant disaster may manifest. Furthermore, the disease rate is expected to noticeably increase because of the implementation of periodic routine thyroid ultrasound screening in Fukushima. This circumstance requires the clarification of the health effect of radiation, protection of health of the prefectural residents for a long term, and continuation of careful health risk communication. In particular, countermeasure based on the scientific evidences and international peer-reviewed processes ${ }^{(30)}$, which utilizes the teachings of Chernobyl experience are required, and simultaneously, the mental care center needs to be established in Fukushima as a countermeasure for negative social and psychological impact.

\section{CONCLUSION}

The declining credibility of the Japanese governmental and official bodies just after the accident may worsen the difficult situation of radiation fear and anxiety. The Fukushima nuclear power plant accident has also severely shuttered public faith in the academic societies in Japan. Therefore, it is important to regain public trust and narrow the gaps in knowledge between the experts and the public on the stochastic effects of lowdose radiation exposure, where a large uncertainty exists, and on public health emergency and radiocontamination from the nuclear disaster, which poses a real problem. So far the only concern is about the increase of thyroid cancer risk among children based on the consequences of Chernobyl. In this respect, it is necessary to establish a system for a long-term followup for all the children because the unprecedented accident really took place in Fukushima. In parallel, more practical preparatory countermeasure planning and actual implementation of radiation emergency medical preparedness before the accident is needed.

From the Fukushima nuclear power plant accident, a variety of problems are exposed in the initial governmental correspondence. In particular, the reexamination of evacuation prepared area, the predistribution of stable iodine pills, interaction with the residents after an accident, the re-examination of public risk communication, and the development of an optimal guideline for the revival and restoration after the accident are necessary. The debating and even contradictory issues of handling subclinical and asymptomatic thyroid cancer should be answered not only in childhood but also in adult patients referring the accumulated data and international standardized guidelines $^{(31-33)}$. We need to carefully analyze the thyroid ultrasound examination data at the standpoint not only of a screening bias and exaggerated discovery rate of thyroid diseases but also of treatment strategy and outcome ${ }^{(34)}$. The appropriate guidelines of clinical management of childhood thyroid cancer need to be established since high detection rate of childhood thyroid diseases has already been reported in other areas in Japan using the same ultrasound screening methodology ${ }^{(35)}$.

Finally, whenever we introduce any countermeasure to the public, especially after the nuclear accident, more profound attention and deliberation should be paid involving any stakeholder to collaborate and cooperate in establishing comprehensive radiation health risk management.

\section{REFERENCES}

1. Pierce, D.A., Shimizu Y., Preston, D.L., Vaeth, M., Mabuchi, K. Studies of the mortality of atomic bomb 


\section{SHORT TITLE}

survivors. report 12, part I. Cancer. 1950-1990. 1996. Radiat Res. 178, AV61-87. (2012).

2. Preston, D.L., Shimizu, Y., Pierce, D.A., Suyama, A., Mabuchi, K. Studies of mortality of atomic bomb survivors. Report 13: solid cancer and noncancer disease mortality: 1950-1997. 2003. Radiat Res. 178, AV146-172. (2012).

3. Yamashita, S. Tenth Warren K. Sinclair keynote addressThe Fukushima nuclear power plant accident and comprehensive health risk management. Health Phys. 106, 166-180. (2014).

4. Suzuki, K., Yamashita, S. Low dose radiation exposure and thyroid carcinogenesis. Jpn J Cin Oncol. 42, 563-568. (2012).

5. Hänscheid, H., Reiners, C., Goulko, G., Luster, M., Schneider-Ludorff M., Buck, A.K., Lassman, M. Facing the Nuclear Threat; thyroid blocking revisit. J Clin Endocrinol Metab. 96, 3511-3516. (2011).

6. Spallek, L., Krille, L., Reiners, C., Schneider, R., Yamashita, S., Zeeb, H. Adverse effects of iodine thyroid blocking; a systemic review. Radiat Prot Dosim. 150, 267277. (2012).

7. Yamashita, S., Suzuki, S. Risk of thyroid cancer after the Fukushima nuclear power plant accident. Resp Invest. 51, 128-133. (2013).

8. Saenko, V., Ivanov, V., Tsyb, A., Bogdanova, T., Tronko, M., Demidchik, Y., Yamashita, S. The Chernobyl accidents and its consequences. Clin Oncol. 23, 234-243. (2011).

9. Kazakov, V.S., Demidchik, E.P., Ashtakhova, L.N. Thyroid cancer after Chernobyl. Nature. 359, 21. (1992).

10. Cardis, E., Kesminiene, A., Ivanov, V., et al. Risk of thyroid cancer after exposure to 131-I in childhood. J Natl Cancer Inst. 97, 724-732. (2005).

11. International Commission on Radiological Protection. United Kingdom: Nonprofit Organization [updated 2013 April 18; cited 2013 April 23]. Available on http://www.icrp.org/.

12. Jacob, P., Kenigsberg, Y., Zvonova, I., et al. Childhood exposure due to the Chernobyl accident and thyroid cancer risk in contaminated areas of Belarus and Russia. $\mathrm{Br} \mathrm{J}$ Cancer. 80, 1461-1469. (1999)

13. Brenner, A.V., Tronko, M.D., Hatch, M., et al. I- 131 dose response for incident thyroid cancers in Ukraine related to the Chernobyl accident. Environ Health Perspect. 119, 933-939. (2011).

14. Ivanov, V.K., Kashcheev, V.V., Chekin, S.Yu., Maksioutov, M.A., Tumanov, K.A., Vlasov, O.K., Shchukina, N. V., Tsyb, A.F. Radiation-epidemiological studies of thyroid cancer incidence in Russia after the Chernobyl accident (estimation of radiation risks, 19912008 follow-up period). Radiat Prot Dosim. 151, 489-499. (2012).

15. Shibata, Y., Yamashita, S., Masyakin, V.B., Panasyuk, G.D., Nagataki, S. 15 years after Chernobyl: new evidence of thyroid cancer. Lancet. 358, 1965-1966. (2001).

16. Demidchik, Y.E., Saenko, V.A., Yamashita, S. Childhood thyroid cancer in Belarus, Russia and Ukraine after Chernobyl and at present. Arq Bras Endocrinol Metabol. 51, 748-762. (2007).

17. Saenko, V., Yamashita, S. Chernobyl thyroid cancer 25 years after: in search of a molecular radiation signature. Hot Thyroidology. HT 8/10. Available on http://www.hotthyroidology.com/. (2010).

18. Takahashi, M., Saenko, V.A., Rogounovitch, T.I., et al. The FOXE1 locus is a major determinant for radiation- related thyroid cancer in Chernobyl. Hum Mol Genet. 19, 2516-2523. (2010).

19. Gudmundsson, J., Sulem, P., Gudbjartsson, D.F., et al. Discovery of common variants associated with low TSH levels and thyroid cancer risk. Nat Genet. 44, 319-322. (2012).

20. Nagataki, S., Takamura, N.. A review of the Fukushima nuclear reactor accident; radiation effects on the thyroid and strategies for prevention. Curr Opin Endocrinol Diabetes Obes. 21, 384-393. (2014)

21. Fukushima Prefecture. Fukushima: Available on http://www.pref.fukushima.lg.jp/sec/21045b/kenkocyosagaiyo.html

22. Yasumura, S., Hosoya, M., Yamashita, S., Kamiya, K., Abe, M., Akashi, M., Kodama, K., Ozasa, K; Fukushima Health Management Survey Group. Study protocol for the Fukushima health managenement survey. J Epidemiol. 22, 375-383. (2012).

23. Tokonami, S., Hosoda, M., Akiba, S., Sorimachi, A., Kashiwakura, I., Balonov, M. Thyroid doses for evacuees from the Fukushima nuclear accident. Sci Rep. 2, 507. (2012).

24. Matsuda, N., Kumagai, A., Ohtsuru, A., Morita, N., Miura, M., Yoshida, M., Kudo, T., Takamura, N. Assessment of internal exposure doses in Fukushima by a whole body counter within one month after the nuclear power plant accident. Radiat Res. 179, 663-668. (2013)

25. World Health Organization. Preliminary dose estimation from the nuclear accident after the 2011 Great East Japan Earthquake and Tsunami. Geneva: 120p. (2012).

26. Prefectural Residents' Health Management Survey By Radiology Health Management Center of Fukushima Medical University. Fukushima:. Available on http://fukushima-mimamori.jp/.

27. The Japan Association of Breast and Thyroid Ultrasound Committee for Thyroidal Terminology's Diagnostic Criteria. 2nd Edition of the Guidebook of Thyroid Ultrasound Diagnosis. Tokyo. (2012).

28. Ozasa, K., Shimizu, Y., Suyama, A., Kasagi, F., Soda, M., Sakata, R., Sugiyama, H., Kodama, K. Studies of the mortality of atomic bomb survivors, Report 14, 1950-2003; an overview of cancer and noncancer diseases. Radiat Res. 177, 229-243. (2012).

29. Furukawa, K., Preston, D., Funamoto, S., Yonehara, S., Ito, M., Tokuoka, S., Sugiyama, H., Soda, M., Ozasa, K., Mabuhi, K. Long-term trend of thyroid cancer risk among Japanese atomic-bomb survivors: 60 years after exposure. Int J Cancer. 132, 1222-1226. (2013).

30. United Nations Scientific Committee on the Effects of Atomic Radiation. Sources and effects of ionizing radiation. UNSCEAR 2008 Report to the General Assembly with Scientific Annexes. Volume II, Scientific Annexes C, D and E. New York: United Nations. (2011).

31. The American Thyroid Association Guideline Taskforce. Management guidelines for patients with thyroid nodules and differentiated thyroid cancer. Thyroid. 16, 109-142. (2006).

32. AACE/AME Task Force on Thyroid Nodules. American Association of Clinical Endocrinologists and Associazione Medici Endocrinologi medical guidelines for clinical practice for the diagnosis and management of thyroid nodules. Endocr Pract. 12, 63-102. (2006). 
33. British Thyroid Association. Royal College of Physicians: Guidelines for the management of thyroid cancer, 2nd edition. (2007)

34. Sugitani, I., Toda, K., Yamada, K., Yamamoto, N., Ikenaga, M., Fujimoto, Y. Three distinctly different kinds of papillary thyroid microcarcinoma should be recognized: our treatment strategies and outcomes. World J Surg. 34, 1222-1231. (2010).

35. Hayashida, N., Imaizumi, M., Shimura, H., et al. Thyroid ultrasound findings in children from three Japanese prefectures; Aomori, Yamanashi and Nagasaki. PLoS One. 8, e83220. (2013). 